\title{
Professionalitätsentwicklung in der allgemeinen, öffentlichen Erwachsenenbildung für Inklusion - ein Systematisierungsversuch der Fortbildungspraxis an Volkshochschulen
}

\author{
Simone Krähling
}

\section{Zusammenfassung}

Die allgemeine, öffentliche Erwachsenenbildung folgt traditionell dem (inklusiven) Prinzip einer Bildung für Alle. Fragen der Bildungsbenachteiligung, Zielgruppendifferenzierung oder Heterogenität werden sowohl in der Wissenschaft als auch in der Praxis virulent. Diskussionen über eine Steigerung der Bildungsteilnahme von Menschen mit Behinderung finden hingegen eher zögerlich statt und werden vermehrt seit der Ratifizierung der UN-Behindertenrechtskonvention in Deutschland geführt. Die Qualifizierung des Personals - häufig konkretisiert in Fortbildungen - stellt dabei ein zentrales Element für eine inklusive Ausrichtung der allgemeinen, öffentlichen Erwachsenenbildung dar. Der Beitrag behandelt die Frage, wie sich die aktuelle Fortbildungspraxis zu Inklusion gestaltet. Auf der Grundlage empirischer Teilergebnisse eines Projekts zur inklusiven Erwachsenenbildung werden Umsetzungsrealitäten vorgestellt und Impulse für eine Professionalitätsentwicklung diskutiert. Die Befunde beziehen sich auf das institutionelle Feld von Volkshochschule und werden am Beispiel der Fortbildungspraxis von Kursleitungen eruiert. Aus einer Organisationsperspektive werden Möglichkeiten und Herausforderungen einer Professionalitätsentwicklung für eine inklusive Erwachsenenbildung - im Besonderen von Lehrpersonal an Volkshochschulen - erfasst.

\section{Schlagworte}

Professionalität, Professionalitätsentwicklung, Erwachsenenbildung, Inklusion, Fortbildungen, Volkshochschulen

\section{Title}

Professional development in public adult education for inclusion - a systematization attempt at further education practice at adult education centres

\begin{abstract}
General public adult education traditionally follows the (inclusive) principle of education for all. Questions of educational disadvantage target group differentiation or heterogeneity are virulent in both science and practice. Discussions about increasing the participation of people with disabilities are rather hesitant and have been held more and more since the ratification of the UN Convention on the Rights of Persons with Disabilities in Germany. The qualification of staff often specified in further training is a key element for the inclusive orientation of general public adult education. The article deals with the question of how the current training practice on inclusion is organised. On the basis of empirical partial results of a project on inclusive adult education implementation realities are presented and impulses for a professional development are discussed. The findings relate to the institutional field of adult education centres and are determined by using the example of the continuing training practice of course leaders. From an
\end{abstract}


organisational perspective opportunities and challenges for professional development for inclusive adult education in particular teaching staff at adult education centres are covered.

\section{Keywords}

Professionalism, professionalism development, adult education, inclusion, further education, adult education centres

\section{Inhaltsverzeichnis}

1. Einleitung

2. Diskurse zur Professionalitätsentwicklung in der allgemeinen, öffentlichen Erwachsenenbildung für Inklusion

2.1. Inklusion in der allgemeinen, öffentlichen Erwachsenenbildung

2.2. Professionalitätsentwicklung in der allgemeinen, öffentlichen Erwachsenenbildung

3. Analyseergebnisse zur Fortbildungspraxis in der allgemeinen, öffentlichen Erwachsenenbildung

3.1. Reaktion auf Fortbildungsbedarfe

3.2. Eigene Fortbildungsangebote

3.3. Kooperative Fortbildungsangebote

3.4. Fortbildungsförderung von Einzelplayern

4. Zusammenfassung und Perspektiven

Literatur

Kontakt

Zitation

\section{Einleitung}

Volkshochschulen (VHS) stellen mit insgesamt 894 Einrichtungen die größten Anbieter allgemeiner, öffentlicher Erwachsenenbildung in Deutschland dar (Reichart, Huntemann \& Lux, 2020, S. 17). Sie gewährleisten flächendeckende, wohnortnahe Weiterbildungsangebote ${ }^{1}$, die allen Bevölkerungsgruppen - in der Regel durch freiwillige Teilnahme - ein breites allgemeinund berufsbildendes Themenspektrum auf unterschiedlichen Leistungsniveaus vorhalten. Nach ihrem Selbstverständnis und gesellschaftspolitischen Auftrag ist Bildung als „[...] Kernvoraussetzung zur Vermeidung und Überwindung von Ausgrenzung und Chance zu umfassender Teilhabe [...]" (Süssmuth \& Eisfeld, 2018, S. 764) definiert; Volkshochschularbeit folgt somit seit jeher dem (inklusiven) Prinzip einer Bildung für Alle. [1]

Die in 2008 durch die Bundesrepublik ratifizierte und in 2009 in Kraft getretene UN-Behindertenrechtskonvention (UN-BRK) verstärkt die Diskurse: Artikel 24 präzisiert in Absatz 5, dass Menschen mit Behinderung durch entsprechende Vorkehrungen einen diskriminierungsfreien und gleichberechtigten Zugang zu Erwachsenenbildung erhalten sollen. Eine Behinderung entsteht dabei aus der Wechselwirkung zwischen der Beeinträchtigung eines Menschen und den Barrieren in seiner Umwelt (Artikel 1 Absatz 2 UN-BRK). Es wird ein inklusiver Gedanke etabliert, der auf die Schaffung struktureller Rahmenbedingungen für eine umfassende Partizipation abzielt (Seitter \& Franz, 2019, S. 3). Für die inklusive Ausrichtung von Einrichtungen der Erwachsenenbildung unter der Perspektive der Förderung gesellschaftlicher Teilhabe stellt sich vor allem die Qualifizierung des Personals heraus (Döbert \& Weishaupt, 2013, S. 7). [2] 
Die allgemeine, öffentliche Erwachsenenbildung weist im Vergleich zum Schulwesen eine verhaltene Auseinandersetzung mit Inklusion auf: Weiterbildungsangebote für Menschen mit Behinderung - insbesondere berufsbezogene - werden zum Großteil in eher separierenden Institutionen der Sozial- und Rehabilitationspädagogik realisiert (Ackermann, 2017, S. 140f.; Autorengruppe Bildungsberichterstattung, 2014, S. 197) oder finden vereinzelt in der allgemeinen, öffentlichen Erwachsenenbildung statt. Individuellen Anliegen von (potenziellen) Teilnehmenden mit Assistenzbedarf wird in der Regel mit kursbezogenen Einzelfalllösungen, weniger systematisch begegnet (Lauber-Pohle, 2019, S. 10). Sowohl in konzeptioneller und handlungspraktischer als auch in empirischer Hinsicht ist ein Desiderat zu verzeichnen - vor allem zu Anforderungen auf organisationaler, professioneller und didaktischer Ebene. [3]

Das Projekt „Qualifizierung für eine inklusive, allgemeine Erwachsenenbildung am Beispiel von Blindheit und Sehbeeinträchtigung - iQ_EB“2 an der Philipps-Universität Marburg setzt daran an und nimmt eine doppelte Perspektive ein: Erstens werden die Bedarfe der hessischen Volkshochschulen als typische Einrichtungen der allgemeinen, öffentlichen Erwachsenenbildung mit Blick auf die Anforderungen an das Personal und die Besonderheiten der unterschiedlichen Programmbereiche erfasst und analysiert. Zweitens werden die Bedarfe zur Umsetzung und Teilhabe an allgemeiner, öffentlicher Erwachsenenbildung von Adressatinnen und Adressaten mit Blindheit oder Sehbeeinträchtigung ${ }^{3}$ als eine Form von Sinnesbeeinträchtigung eruiert. Im vorliegenden Beitrag wird der Begriff der Sehbeeinträchtigung genutzt, da dieser auf die höchst unterschiedlichen Formen und Grade (angeboren oder erworben, stabil oder fortschreitend, singulär oder komplex in Verbindung mit weiteren Beeinträchtigungen) sowie die je unterschiedlichen Anforderungen an die Umwelt verweist. Die spezifische Aneignung von und Auseinandersetzung mit der materialen und sozialen Umwelt erfordern dabei differenzierte pädagogische Implikationen (Walthes, 2014, S. 18f.). [4]

Die Forschungsergebnisse des genannten Projekts münden in Kooperation und Abstimmung mit den Verbänden des Praxisfeldes ${ }^{4}$ in zwei Fortbildungsreihen für eine inklusive Erwachsenenbildung, die an die unterschiedlichen VHS-Personalgruppen der Einrichtungsleitung bzw. Planung und Lehre gerichtet sind. Eine wesentliche Grundlage für die Konzipierung der Veranstaltungen stellen nicht nur die artikulierten Fortbildungsbedarfe des Personals, sondern auch die aktuelle Fortbildungspraxis zu Inklusion an den untersuchten Volkshochschulen dar. [5]

Der vorliegende Artikel widmet sich einem Systematisierungsversuch dieser und fokussiert sich auf die Fortbildungsmöglichkeiten für das Lehrpersonal. Diesem kommt eine Schlüsselrolle zu, da es für die Angebotsgestaltung - einem der Kernprozesse erwachsenenpädagogischen Handelns - verantwortlich ist und sich im Direktkontakt mit Kursteilnehmenden sowie möglichen Assistenzbedarfen befindet. Zentrale Frage ist, welche Fortbildungsrealitäten sich für eine inklusive Erwachsenenbildung in den Untersuchungseinrichtungen abzeichnen. Fortbildung wird hier als essentieller Bestandteil von Professionalitätsentwicklung in der allgemeinen, öffentlichen Erwachsenenbildung verstanden und aus einer Organisationsperspektive heraus beleuchtet. [6]

Zunächst wird in das Thema der Professionalitätsentwicklung in der allgemeinen, öffentlichen Erwachsenenbildung gegenstandstheoretisch eingeführt, in den Kontext von Inklusion gesetzt und mit Fokus auf Fortbildungen Merkmale des Institutionalfeldes herausgearbeitet (Kapitel 2). Im Anschluss werden empirische Teilergebnisse zur aktuellen Fortbildungspraxis für Inklusion in der hessischen VHS-Landschaft anhand von vier Dimensionen vorgestellt (Kapitel 3). Hinführend wird das methodische Vorgehen innerhalb des genannten Projekts und die Herleitung seiner Relevanz für die Beantwortung der zentralen Frage skizziert. Die Analyseergebnisse werden in einem Fazit zusammengefasst und hinsichtlich ihrer Perspektiven diskutiert (Kapitel 4). [7] 


\section{Diskurse zur Professionalitätsentwicklung in der allgemeinen, öffentlichen Erwachsenenbildung für Inklusion}

Im Folgenden werden unterschiedliche Diskurslinien zur Professionalitätsentwicklung für Inklusion skizziert: Es werden der aktuelle Stand der Inklusionsdebatte in der allgemeinen, öffentlichen Erwachsenenbildung vorgestellt (Kapitel 2.1) sowie Ableitungen von Implikationen und Herausforderungen für eine Professionalitätsentwicklung des VHS-Personals getroffen (Kapitel 2.2). [8]

\subsection{Inklusion in der allgemeinen, öffentlichen Erwachsenenbildung}

In den letzten Jahren hat sich der Diskurs zu einer inklusiven Erwachsenenbildung verstärkt: Neben theoretischen Zugängen (Burtscher, Ditschek, Ackermann, Kil \& Kronauer, 2013) sowie der Bedeutung von Kooperationen (Ackermann, Burtscher, Ditschek \& Schlummer, 2012), allgemeiner Rahmenbedingungen (Babilon, 2018) oder der Didaktik (Furrer, 2013) findet auch eine Auseinandersetzung in einschlägigen Fachzeitschriften der Erwachsenenbildung statt, z.B. in den Hessischen Blättern für Volksbildung (1/2019). Im Kontext von Vielfalt und Heterogenität als konstitutive Bestandteile allgemeiner, öffentlicher Erwachsenenbildung kommt Inklusion ein hoher Stellenwert zu. Ziel ist, „[...] Institutionen so zu gestalten, dass alle Menschen unterrichtet werden und lernen können, indem sie gewürdigt werden, wie sie sind, partizipieren und ihr Potenzial entfalten können" (Hirschberg \& Lindmeier, 2013, S. 39). Inklusive Bildung ist somit als ein Konzept, Modell und Prozess für eine selbstverständliche Teilhabe von Menschen mit Behinderung nach dem Prinzip einer Bildung für Alle (Babilon, 2018, S. 7) und institutionelle Querschnittsaufgabe definiert (Babilon, Weiß \& Brandt, 2019, S. 15f.). [9]

Im Institutionalfeld wird häufig ein weites Inklusionsverständnis angewendet, das sich vorrangig traditionellen VHS-Zielgruppen wie Menschen mit Migrationshintergrund, Langzeitarbeitslosen, Analphabetinnen und Analphabeten oder Älteren widmet (Seitter \& Franz, 2019, S. 4). Zielgruppenorientierung ${ }^{5}$ konkretisiert sich in Spezialkursen mit Fokus auf der Integration benachteiligter Gruppen. Eine allgemeine Zielgruppenansprache von Menschen mit Behinderung erscheint aufgrund der ausgeprägten Heterogenität des Personenkreises nicht sinnvoll; vielmehr ist eine Differenzierung in unterschiedliche Gruppen und Grade von Beeinträchtigungen (Babilon, 2018, S. 65) oder ein wachsender Assistenzbedarf innerhalb anderer Zielgruppen, z.B. Ältere, zu beobachten (Lauber-Pohle, 2019, S. 15). So liegt im Berichtsjahr 2018 der VHS-Statistik der Kursanteil der Personen über 65 Jahren bei insgesamt 18,4 Prozent (Reichart et al., 2020, S. 88). Im Zuge der demografischen Entwicklungen steigt perspektivisch die Anzahl von Menschen mit einer altersbedingten Beeinträchtigung (Deutsches Zentrum für Altersfragen, 2016, S. 23f.) wie es exemplarisch Korb et al. (2014) für altersbedingte Sehbeeinträchtigungen und einer implizierten Einschränkung bzw. einem implizierten Verlust der Lesefähigkeit festhält. [10]

Zusammenfassend bewegen sich die Diskurse zur Professionalitätsentwicklung in der allgemeinen, öffentlichen Erwachsenenbildung für Inklusion zwischen dem multikomplexen Anspruch gelebter Vielfalt und Heterogenität im Sinne einer umfassenden Öffnung sowie der Kategorisierung in und Exklusivität von Gruppen. Letzteres schafft vielfach erst eine Zugangsmöglichkeit für Menschen mit einem spezifischen Assistenzbedarf (Babilon et al., 2019, S. 14f.). Die Münchner Erklärung zur Inklusion und öffentlich verantworteten Erwachsenenbildung (2015) hält fest, dass auf dem Weg zur inklusiven Ausrichtung von Einrichtungen Angebote, Prozesse und Strukturen (fort-)entwickelt werden müssten. Dies schließt auch „inklusionsorientierte Weiterbildungen für alle in der Erwachsenenbildung Tätigen" (Münchner Volkshochschule, 2015) ein. Aufgrund ihrer Schlüsselfunktion kommt vor allem der Sensibilisierung sowie entsprechenden Aus- und Fortbildung der Lehrkräfte eine zentrale Bedeutung zu. Eine Professionalitätsentwicklung impliziert „neben Formen der behinderungsspezifischen Kursvorbereitung (didaktisches Design, Unterrichtsmaterialien, Prüfungsformen) vielfältige Varianten inklusionsorientierter didaktischer Möglichkeiten [...] wie Binnendifferenzierung, variable Lerntempi oder unterschiedliche Darbietungs- und Erfassungsmodi der Lerngegenstände" (Lauber-Pohle \& Seitter, 2020, Abs. 12). [11] 


\title{
2.2. Professionalitätsentwicklung in der allgemeinen, öffentlichen Erwachsenenbildung
}

Volkshochschulen als typische Einrichtungen der allgemeinen, öffentlichen Erwachsenenbildung unterliegen in den letzten Jahrzehnten - durch die Auseinandersetzung mit und Anpassung an gesellschaftlichen und beruflichen Megatrends - weitreichenden Modifikationen ihres Profils und können als lernende Organisationen ${ }^{6}$ definiert werden. Die Veränderungen beziehen sich nicht nur auf die Angebotsplanung oder das Einrichtungsmanagement, sondern auch auf eine Professionalitätsentwicklung der in der allgemeinen, öffentlichen Erwachsenenbildung Tätigen (Süssmuth \& Eisfeld, 2018, S. 770): [12]
„Die Frage nach aktuellen und zukünftigen Herausforderungen in der Weiterbildung, aber auch nach Aufgaben und Tätigkeiten der Weiterbildner/innen sowie Fragen nach der Qualifikation und dem Fortbildungsangebot geben in diesem Kontext Aufschluss über thematische Trends und Rahmenbedingungen der Fortbildung von Weiterbildner/ innen“ (Hippel \& Tippelt, 2009, S. 12). [13]

Im Zuge dessen bilden Fortbildungen ein zentrales Element von Professionalitätsentwicklung und können die Basis für die Realisierung einer inklusiven Erwachsenenbildung darstellen. Professionalität, hier als "gekonnte Beruflichkeit, als Indikator für qualitativ hochwertige Arbeit“ (Nittel, 2000, S. 15) definiert, konkretisiert sich auf der Ebene des beruflichen Handelns und ist unabhängig vom Einstellungsverhältnis. Sie prägt in einem entscheidenden Maße die Angebotsqualität (Kraft, Seitter \& Kollewe, 2009, S. 13). [14]

Der aktuelle Bildungsbericht (2018) fasst exemplarisch für den Gesamtsektor Weiterbildung eine überdurchschnittlich hohe Weiterbildungsbeteiligung zusammen und nimmt auch Umsetzungsrealitäten von Fortbildung der Institution Volkshochschule in den Blick (Autorengruppe Bildungsberichterstattung, 2018, Abschnitt G3). Der Deutsche Volkshochschul-Verband (DVV) forciert seit den 1970er Jahren - auf Grundlage der Erwachsenenbildungsgesetze in den Ländern - die Verberuflichung und Qualifizierung des Personals (Gieseke, 2018, S. 1062). Die rund 197.000 neben- und freiberuflich Lehrenden, die vorrangig für die Gestaltung von Kursen oder Vorträgen in Einzelveranstaltungen verantwortlich sind, bilden dabei mit Abstand die größte Personalgruppe an Volkshochschulen - gegenüber den hauptamtlichen Tätigkeitsverhältnissen von insgesamt 9.500 Vollzeitäquivalenten (Reichart et al., 2020, S. 14): [15]

\begin{abstract}
„Als Multiplikator von Ideen, Inhalten und Identifizierung sowie als personifiziertes Bindeglied zwischen den Teilnehmenden und der Einrichtung erfüllen die (meist freiberuflichen) Dozenten eine wichtige Aufgabe. Allerdings hat die Einrichtung ihrerseits keinen echten Zugriff auf die Einstellung bzw. Loyalität der meist freiberuflich tätigen Lehrenden gegenüber der Institution“ (Möller, 2011, S. 80). [16]
\end{abstract}

Dies verweist auf das Merkmal der doppelten Professions- und Tätigkeitsausrichtung des VHSPersonals: Leitung und Planung zeichnen sich in der Regel durch Festanstellungen mit formaler Organisationszugehörigkeit, Lehrende durch neben- und freiberufliche Beschäftigungsverhältnisse mit geringem Regulierungsgrad aus. Während ein Großteil der Weiterbildungsangebote von Kursleitungen gestaltet wird, die sich maßgeblich durch Fachkompetenzen für ihre Tätigkeit qualifizieren, besitzt das hauptberufliche Leitungs- und Planungspersonal vornehmlich pädagogisch-akademische Berufsabschlüsse (Martin et al., 2016, S. 117). [17]

Das Lehrpersonal partizipiert eher an kürzeren Fortbildungsformaten und finanziert diese häufig selbst (Autorengruppe Bildungsberichterstattung, 2018, S. 182f.). „Problematisch ist, dass die Gruppe der nebenberuflichen Mitarbeiterinnen und Mitarbeiter sehr heterogen in Bezug auf Motivation, pädagogische Qualifikation, Rollenauffassung und Eingangsvoraussetzungen ist" (Harmeier, 2009, S. 22). Aufgrund knapper Ressourcen von Volkshochschulen - sowohl in finanzieller als auch in personeller Hinsicht - gestalten sich die Fortbildungsmöglichkeiten vergleichsweise prekär. Die allgemeine, öffentliche Erwachsenenbildung steht daher vor der Herausforderung, neuen Lernfeldern und den implizierten Anforderungen an Qualifikation unter 
Berücksichtigung der Spezifika des Institutionalfeldes zu begegnen. Welche Handlungsoptionen sich im Kontext von Inklusion herauskristallisieren lassen, soll im Folgenden anhand der aktuellen Fortbildungspraxis erörtert werden. [18]

\title{
3. Analyseergebnisse zur Fortbildungspraxis in der allgemeinen, öffentlichen Erwachsenenbildung
}

Zur Beantwortung der zentralen Frage, welche Fortbildungsrealitäten sich für eine inklusive Erwachsenenbildung in der Institution Volkshochschule abzeichnen, kann das Datenmaterial des Projekts "Qualifizierung für eine inklusive, allgemeine Erwachsenenbildung am Beispiel von Blindheit und Sehbeeinträchtigung - iQ_EB" an der Philipps-Universität Marburg beitragen. Das Vorhaben fokussiert sich auf die Bedarfe und Erfahrungen zur Umsetzung einer inklusiven Erwachsenenbildung von Volkshochschulen sowie von Menschen mit Blindheit oder Sehbeeinträchtigung. Das Projekt verwendet in der Datenerhebung und -auswertung ein komplexes Mixed-Methods-Design bestehend aus quantitativen und qualitativen Methoden wie Literaturund Dokumentenanalysen, halbstandardisierte Leitfadeninterviews, Online-Befragungen, Fallanalysen, inhalts- und sequenzanalytische Auswertungsverfahren oder deskriptive Statistik. Aufgrund des weitgehenden Forschungsdesiderats weisen die empirischen Projektergebnisse einen explorativen Charakter auf. Sie bergen das Potenzial, auf nationaler und internationaler Ebene unter der Berücksichtigung weiterer Segmente der Weiterbildung und weiterer Formen von Beeinträchtigung fortgeführt zu werden. Das Vorhaben besitzt nicht nur eine empirische, sondern auch konzeptionelle Ausrichtung und zielt auf die Entwicklung von zwei Fortbildungsreihen für die unterschiedlichen VHS-Personalgruppen der Einrichtungsleitung bzw. Planung und Lehre ab (Kapitel 1). [19]

Fortbildung als ein zentrales Element von Professionalitätsentwicklung für Inklusion tritt somit im vorliegenden Artikel aus zwei Perspektiven hervor: Erstens als Forschungsgegenstand in Form einer Analyse empirischer Teilergebnisse zu aktuellen Umsetzungsrealitäten, zweitens als handlungspraktischer Beitrag in Form der Konzeptionierung von Fortbildungsmodulen für eine inklusive Erwachsenenbildung. Dies zeigt sich an Seitters (2009) Verständnis von Professionalitätsentwicklung anschlussfähig: [20]

\begin{abstract}
„Professionalitätsentwicklung ist auf der einen Seite eine empirisch abgesicherte Beobachtung von Veränderungsprozessen, die sich gegenwärtig im Feld der Weiterbildung vollziehen. Ihre Beschreibung und Analyse ist insofern eine Forschungsaufgabe. Auf der anderen Seite meint Professionalitätsentwicklung jedoch auch einen feldbezogenen Entwicklungsimpetus, der eine bestehende Praxis optimieren möchte. Ihre Umsetzung und Beförderung ist insofern eine handlungspraktische Gestaltungsaufgabe" (Seitter, 2009, S. 13, Hervorhebung im Original). [21]
\end{abstract}

Die empirischen Teilergebnisse, die im vorliegenden Beitrag dargestellt werden, gründen sich auf qualitative, halbstandardisierte Leitfadeninterviews mit Leitungen der Volkshochschulen in Hessen $(n=29)$ zu ihren Erfahrungen mit Inklusion, den Qualifikationsbedarfen für sich und ihr Personal sowie den Entwicklungsperspektiven der Gesamtorganisation. Die Leitungskräfte bilden die hessische Grundgesamtheit von 33 Einrichtungen ab (32 VHS plus Hessische HeimVHS Burg Fürsteneck) und waren daher klar identifizierbar. Der Erhebungsphase folgte mittels der inhaltlich strukturierenden qualitativen Inhaltsanalyse eine Querschnittsauswertung (Kuckartz, 2018). [22]

Das Datenmaterial spiegelt die Sicht der hauptberuflichen VHS-Leitungen wider, die ihre Organisation repräsentieren und im Sinne der Gesamtverantwortlichkeit die Fortbildungsrealitäten des Lehrpersonals in einem entscheidenden Maße steuern. Die Kategorie Personalqualifizierung - differenziert nach den unterschiedlichen VHS-Personalgruppen - versammelt Leitungsaussagen sowohl zur aktuellen Fortbildungspraxis der Kursleitungen in der jeweiligen Einrichtung (zu Inklusion) als auch Ideen dazu. Einige Gespräche erfolgten unter der Beteiligung hauptamtlicher pädagogischer Mitarbeitenden (HPM), die aufgrund eines Inklusionsbezugs von ihren Vorgesetzten zu den Interviews eingeladen wurden. [23] 
Im Folgenden werden vier Dimensionen der Fortbildungspraxis (zu Inklusion) an Volkshochschulen voneinander differenziert und durch ausgewählte, prägnante Sequenzen nah am Datenmaterial erläutert. Die Systematisierung ist als eine empirische Annäherung an die Thematik zu verstehen und könnte durch weiterführende Untersuchungen modifiziert und/oder ergänzt werden. [24]

\subsection{Reaktion auf Fortbildungsbedarfe}

Ein zentrales Analyseergebnis ist, dass Fortbildungsbedarfe von Kursleitungen bei Umsetzungsfragen von Inklusion virulent werden. Diese bilden sich anlassbezogen und zumeist problemzentriert ab: [25]

„Wir hatten jetzt ein Problem mit einem traumatisierten Teilnehmer, der hat tatsächlich einen großen, ja, ein großes Problem im Kurs, also hat die Kurssituation gestört. Und danach, erst nach diesem Anlass, [...] haben dann mehrere Kursleitende in dem Treffen unter den Kursleitenden [...] gesagt: Ja, wir wollen den Tageskurs zum Thema Umgang mit traumatisierten Teilnehmern" (B28_Leitung, Abs. 135). [26]

Die Absprachen zu Fortbildungen finden in der Regel zwischen den Lehrpersonen und ihrer zugeordneten Programmbereichsleitung statt, die sich dafür verantwortlich zeichnet: „Ansonsten in den Programmbereichen, genau, die Planung und auch die konkreten Absprachen mit den Kursleitungen, das ist dann im gewissen Sinne separiert“ (B9_Leitung, Abs. 45). Die monetäre (Teil-)Förderung besuchter Veranstaltungen bildet dabei eine gängige Umsetzungspraxis. Darauf verweist - unter Verwendung von Begriffen wie Bezuschussung, Kostenübernahme oder Weiterbildungsetat - ein Großteil der Befragten. Die Ausgestaltung vollzieht sich in der Regel getrennt von den hauptberuflichen Förderhaushalten und kann variieren: [27]

„Genau, also jede Volkshochschule, ich kann mir jetzt nicht vorstellen, dass eine das anders macht, haben wir einen gewissen Etat für Fortbildungen und wir übernehmen die Kosten nicht komplett, sondern wir bezuschussen das, also das ist dann immer so Abmessungssache und Absprachesache mit den einzelnen Kursleitungen, wie hoch dann diese Bezuschussung stattfindet. Und im Rahmen dieses Etats finden dann die Fortbildungen statt“ (B20_Leitung, Abs. 115). [28]

Die (ko-)finanzierten Veranstaltungen haben entweder einen direkten Bezug zum Fachthema der Kursleitungen oder fokussieren sich auf Inhalte der Kursdidaktik. Besucht werden vorrangig Fortbildungsangebote, die eher wohnortnah bei regionalen Trägern stattfinden. Darunter können auch bundeslandweite, verbandliche Veranstaltungen gefasst werden, die ein breites Repertoire an Themen für eine erwachsenenpädagogische Qualifizierung anbieten. Dabei besteht bei erhöhtem Interesse die Option einer dezentralen Umsetzung in den jeweiligen Volkshochschulen. Eine Vielzahl der Untersuchungseinrichtungen bewirbt die Möglichkeit einer monetären (Teil-)Förderung von Fortbildungen bei der Aufnahme einer Lehrtätigkeit z.B. im Rahmen eines Erstgesprächs mit der jeweiligen Programmbereichsleitung oder von Einführungsveranstaltungen für neues Lehrpersonal. Darüber hinaus berichtet eine Gesprächsperson: [29]

\footnotetext{
„Also der Weiterbildungsetat wird ganz gut in Anspruch genommen, aber meistens gar nicht ganz voll ausgeschöpft und nicht aus mangelndem Interesse. Ich schilderte ja eben, wir haben eben viele, die neben ihrem Beruf dann abends den Kurs bei der VHS dann noch machen, aber neben diesem Kurs dann noch eine Weiterbildung ist halt schwierig“" (B11_Leitung, Abs. 235). [30]
}

Der geringe Regulierungsgrad der neben- und freiberuflichen Kursleitungen wird maßgeblich im Kontext von Fortbildungen diskutiert - mancherorts auch unter dem Aspekt der Scheinselbstständigkeit. Mit Blick auf Inklusion erläutern einige Befragte, bedarf es neben einem eher reaktiven, situativen Verhalten auf Einzelanfragen weiterführende proaktive Formen einer Bedarfsdeckung bzw. Bedürfnisweckung. [31] 


\subsection{Eigene Fortbildungsangebote}

Eine weitere etablierte Form, Fortbildungswünschen von Kursleitungen für Inklusion entgegenzukommen oder das Interesse dafür zu wecken, stellt die Konzeptionierung und Umsetzung eigener Veranstaltungen dar. Diese greifen sowohl geäußerte Fortbildungsbedarfe von Lehrkräften als auch Qualifikationsimpulse von der jeweiligen Volkshochschule bzw. den Programmbereichsleitungen auf. Letzteres konkretisiert sich darin, Handlungsdefiziten von Kursleitungen, die sie selbst (noch) nicht identifizieren, durch die Realisierung entsprechender Maßnahmen zu begegnen. Bezugnehmend auf ein Spezialangebot für Menschen mit Behinderung entstand in einer Volkshochschule folgender Fortbildungsbedarf: [32]

„Und dann sind natürlich Angebote, also in der Kursleiterfortbildung ein Angebot zum Beispiel, wie kann ich denn Dinge in einfache Sprache übersetzen, ja. Also jetzt gerade im Kontext mit [Einrichtung der Selbsthilfe] ist das entstanden, die verstehen einfach unsere Seminarausschreibungen nicht, ja, können sie nicht verstehen [...]“" (B16_HPM, Abs. 123). [33]

Die Veranstaltungen sind zumeist kostenfrei und werden (pro Semester) aktiv beworben. Entweder übernehmen die hauptberuflichen Mitarbeitenden die Angebotsgestaltung oder es werden externe Referentinnen und Referenten mit einschlägiger Expertise akquiriert. Ein Großteil der Befragten weist auf eine praxisnahe und erfahrungsorientierte Angebotsumsetzung hin, die sich in einem Mehrwert für das neben- und freiberufliche Lehrpersonal manifestiert: [34]

„Insofern ist es, glaube ich, auch eine Aufgabe bei einer Fortbildung, dass von der Themensetzung her runterzubrechen auf das, was die Praxis betrifft und was die Kursleiter und Kursleiterinnen dann auch suchen, also das scheint mir ganz wichtig, das ist einfach so eine Erfahrung, die wir gemacht haben" (B19_Leitung, Abs. 117). [35]

Die Veranstaltungen werden nicht nur den Kursleitungen der jeweiligen Volkshochschule angeboten, sondern auch für Interessierte wie Angehörige von Menschen mit Behinderung geöffnet. Daneben gibt es Angebote zu Inklusionsthemen im regulären Programm, die wiederum den Kursleitungen als Fortbildungsmöglichkeiten dienen können. Die Veranstaltungen können sich auf einen Programmbereich oder auf übergreifende Inhalte zur Bewältigung der Handlungspraxis beziehen: [36]

„Wir hatten darüber hinaus, [...] machen wir Veranstaltungsreihen für Kursleiterinnen und Kursleiter, um sie nicht nur zu sensibilisieren, sondern speziell Angebote für Menschen mit Behinderungen anzubieten. Also was kann ich als Kursleitung zum Beispiel im kulturellen Bereich für Menschen mit Behinderung speziell konzipieren“ (F3_Leitung, Abs. 7). [37]

Häufig nennen die Befragten, ihre Kursleitungen mittels einer Grundqualifizierung auf ihre Tätigkeit vorbereiten zu wollen. Umgang mit Heterogenität in seinen Dimensionen bildet dabei eines der zentralen Themen und wird im Kontext von Inklusion angeführt: [38]

„Umgang mit heterogenen Lerngruppen. Und wie heterogen die dann sind, ich meine, die sind immer heterogen, der eine ist so schnell, der andere ist so schnell, selbst unter, weiß nicht, heteronormativen und neurotypischen Kursteilnehmern sind die Unterschiede schon riesig. [...] Und da kommt vielleicht jemand mit dem Rollstuhl dazu, kommt jemand mit einer Form von Autismus dazu. Das ist dann sozusagen, erweitert nur dieses Spektrum und da wird man eben niemals, [...] wird man niemals alle möglichen Fälle irgendwo einfangen können und jemanden darauf vorbereiten können, sondern es geht darum, Leute fit zu machen dafür, für heterogene Lerngruppen“ (B5_HPM, Abs. 62). [39]

Festzuhalten ist, dass eine Vielzahl der Untersuchungseinrichtungen ihrem Lehrpersonal eigens konzipierte Fortbildungsangebote bietet, die - in unterschiedlichem Ausmaß - kontinuierlich stattfinden und sich an den aktuellen Qualifikationsbedarfen ausrichten. Im Zuge von Inklusion fällt der Blick wiederholt auf das Planungspersonal: Einige VHS-Leitungen berichten von 
Fortbildungsmodi, um die HPM für die Begleitung, Beratung und Motivation ihrer jeweiligen Lehrenden zu qualifizieren. Zudem wird das Potenzial kombinierter Angebote für das Planungsund Lehrpersonal gehoben. [40]

\subsection{Kooperative Fortbildungsangebote}

Die Entwicklung und Realisierung von Fortbildungen mit (regionalen) Kooperationseinrichtungen stellt eine weitere Umsetzungspraxis dar. Anlass bilden entweder konkrete Bedarfe oder die Bearbeitung übergreifender Themen. Das Datenmaterial zeigt auf, dass Volkshochschulen, also Institutionen mit gleichem Bildungsauftrag, gemeinsame Veranstaltungen ausrichten können (horizontale Perspektive) ${ }^{7}$ Eine Gesprächsperson berichtet, sich mit umliegenden Volkshochschulen hinsichtlich ihrer Fortbildungsbedarfe zu vernetzen und in Federführung entsprechende Formate zu organisieren. Folgende Idee konkretisiert sie im Kontext von Inklusion: [41]

„[...] also [VHS in Mittelhessen 1] hat ähnlichen Bedarf bekundet, [VHS in Mittelhessen 2] war dann auch interessiert an dieser Fortbildung, wie die, für Kursleitungen und für Verwaltungsmitarbeiter, nicht konkret auf Inklusion, aber das werde ich definitiv dann auch organisieren, dass die Kursleitungen besser mit körperlichen Beeinträchtigungen im Kurs dann auch zurechtkommen" (B20_Leitung, Abs. 123). [42]

Vereinzelt werden Fortbildungen im Rahmen der Zusammenarbeit mit Einrichtungen anderer Bildungsbereiche thematisiert oder das Potenzial einer solchen Kooperationsausrichtung gehoben (vertikale Perspektive). Vornehmlich werden Hochschulen (in der Region) genannt, die mit inrer Fachexpertise und einer entsprechenden Didaktik aufwarten. Weiterführend finden kooperative Fortbildungen mit Institutionen außerhalb des Bildungsbereichs statt (diagonale Perspektive); darunter fallen Ämter und Verwaltungen, Betriebe oder Sozial- und Kultureinrichtungen. Eine Leitungskraft verweist auf eine formale Kooperation mit einer privaten Institution, die den VHS-Kursleitungen eine kostenfreie bzw. kostenreduzierte Fortbildungsteilnahme ermöglicht: [43]

„Ja, genau, und dort haben wir richtig einen Kooperationsvertrag, der noch weitergeht als mit [Sozialer Unternehmensgruppe], weil [der private Klinikbetreiber] bietet ja eigene Weiterbildungen an und aufgrund dieses Vertrages können dann unsere Dozenten die meisten Weiterbildungen kostenfrei besuchen bei denen oder kostenreduziert" (B11_Leitung, Abs. 97). [44]

Eine enge Verzahnung mit Ämtern und Verwaltungen zeigt sich vor allem bei Volkshochschulen mit öffentlich-rechtlichem Status und kommunaler Ausrichtung. Die Nutzbarmachung der jeweiligen Expertise kann in Fortbildungen aufgehen: [45]

„Und diese, der damalige Behindertenbeauftragte hat dann auch für uns speziell eine Fortbildung für Kursleitungen zum Thema Umgang mit Menschen mit psychischen Beeinträchtigungen angeboten. Also wenn wir da so einen Bedarf sehen, versuchen wir auch, etwas zu organisieren“ (F2_Leitung, Abs. 261). [46]

Die Gesamtschau der skizzierten Analyseergebnisse zeigt, dass im Bereich der vielfältigen Kooperationsbeziehungen in der allgemeinen, öffentlichen Erwachsenenbildung auch der Fortbildungskontext eine Ebene der Zusammenarbeit bilden kann. Fortbildung stellt dabei weniger einen ausschließlichen Zweck, sondern vielmehr ein weiteres Handlungsfeld dar. Umfang und Intensität können sich stark im Institutionalfeld differenzieren: Punktuelle und zumeist bedarfsbezogene Fortbildungen überwiegen jedoch gegenüber regelmäßigen Veranstaltungen. Die Analysekategorie lässt insbesondere zwischen Volkshochschulen einer Region eine verstärkte Vernetzung erkennen. Eine Gesprächsperson erzählt: [47]

„[...] wir werden dann auch die Kursleitenden von uns, die bestimmte Sachen, die werden das hier schulen, die werden das aber auch in den anderen nordhessischen Landkreisen anbieten und genauso werden die Nordhessen rumreisen, das heißt, da 
muss nicht jeder die tolle Idee zu diesem Bereich haben, sondern wir werden diese Ideen in Fortbildungen auch austauschen“ (B14_Leitung, Abs. 69). [48]

Der Regionsbezug stellt sich als eine zentrale Komponente heraus und konkretisiert sich in der Entwicklung und Realisierung unterschiedlicher Fortbildungsformate wie dem Informations- und Erfahrungsaustausch zwischen den Kursleitungen umliegender Volkshochschulen. Eine Verortung von Inklusion in kooperativen Veranstaltungen bündelt somit Ressourcen und kann sowohl übergreifend als auch fachspezifisch umgesetzt werden. [49]

\title{
3.4. Fortbildungsförderung von Einzelplayern
}

Die Analyse zeigt, dass Volkshochschulen auch explizit Fortbildungen einzelner Kursleitungen für Inklusion unterstützen. Der erste Schritt bildet die Identifikation von Lehrenden, die in ihrem Kursgeschehen mit konkreten Assistenzbedarfen konfrontiert sind bzw. waren: „Und vielleicht auch ein Erfahrungsaustausch von Kursleitenden, dass die merken, aha, andere haben das schon gemacht, die haben das so gemacht, das geht offensichtlich, es ist kein Einzelfall" (B24_HPM, Abs. 178). Eine weitere Form der Identifikation stellt die Ansprache von Kursleitungen dar, die einen (berufs-)biografischen Inklusionsbezug aufweisen: [50]

\begin{abstract}
„Wir wollen verschiedene Kursleiterinnen und Kursleiter identifizieren, die wir dann auch ansprechen würden, die entweder schon einen sonderpädagogischen Background haben oder die ein besonderes Interesse auch an der Zielgruppe haben, und würden gerne mit diesen Akteuren in eine gemeinsame Qualitätsentwicklung, gemeinsame Konzeptentwicklung gehen" (F1_Leitung, Abs. 77). [51]
\end{abstract}

Festzuhalten ist, dass Lehrende mit einer generellen Affinität und Motivation, sich (neuen) Themen zu stellen und ihre Angebotsgestaltung (weiter) zu entwickeln, eine große Bereitschaft signalisieren, sich für Inklusion fortzubilden. Die gemeinsame Bearbeitung pädagogischer Prozesse kann somit eine Qualitätsentwicklung und -sicherung befördern. Eine Leitungskraft stellt einen exemplarischen Ablauf in ihrer Einrichtung vor: [52]
„Im Moment geht es mir noch ein bisschen darum, [...] ich habe Kursleitende, die haben Interesse, ein konkretes Angebot inklusiv zu gestalten und die würde ich gerne dabei oder in einer Gruppe unterstützen. So, das ist das eine, und dann auch zu klären, was heißt für die inklusiv konkret, oder wenn man jetzt schon in Kooperation mit einem Träger wie der [Einrichtung der Selbsthilfe] ist, dann ist es schon, ist die Zielgruppe relativ klar, dann kann man sich darauf vorbereiten und ganz konkret auch an dem eigenen Angebot arbeiten. Das ist so das, wo einfach auch Austauschbedarf ist und Nachfragebedarf, wo es sicher für uns auch gut ist, auf die Kompetenz der Kooperationspartner zurückgreifen zu können. Also was bestimmte Krankheits- oder Beeinträchtigungsbilder auch betrifft. Und das ist so das eine auf der Angebotsebene“ (B9_Leitung, Abs. 87). [53]

Obiges Zitat beinhaltet, durch die Förderung einzelner Kursleitungen (zunächst) Grundkenntnisse von Inklusion zu vermitteln bzw. ein gemeinsames Verständnis davon herzustellen (Kapitel 3.2). Zudem wird die Bedeutung von Kooperationseinrichtungen für die Qualifikation des Lehrpersonals betont: Ihre Fach- und Feldexpertise kann zum einen der Wissensvermittlung, zum anderen der Angebotskonzeption dienen. Partnerinstitutionen können dabei in Form kooperativer Fortbildungsangebote aktiv in die Professionalitätsentwicklung der Kursleitungen eingebunden sein (Kapitel 3.3). Eine exemplarische Facette davon kann die Schaffung von Erfahrungsräumen im Sinne von Hospitationen bilden. Eine Gesprächsperson schildert: [54]

„Es gab dann explizit eine Kursleiterin, die aufgrund ihrer Vorbildung sich das auch zugetraut hat und die bereits Erfahrung auch auf privater Ebene mit Menschen mit Behinderung hatte, die dann sagte: ,Ich würde das gerne versuchen'. Die haben wir ein bisschen, die haben wir unterstützt, indem wir sie haben hospitieren lassen bei anderen Trägern, die solche Angebote bereits haben. Dann haben wir gemeinsam ein kleines Konzept entwickelt und sie hat das in der Praxis umgesetzt und dann gab es eben auch ein Feedback, wie es gelaufen ist" (B27_HPM, Abs. 80). [55] 
Die explizite Förderung bildungsaffiner Kursleitungen, „[...] die in Anführungszeichen auch als Pioniere dann arbeiten [...]" (F1_Leitung, Abs. 79), beinhaltet in einer Vielzahl der Interviews die Frage, wie eine Multiplikatorenwirkung erreicht und das individuelle Wissen auf die Gesamtgruppe des Lehrpersonals übertragen werden kann. Eine Möglichkeit besteht darin, dass die fortgebildeten Lehrenden im Bedarfsfall als Ansprechpersonen zur Verfügung stehen. Weiterführend nennen einige Befragte Techniken der kollegialen Fallberatung. Auch können Kursleitungsvertretungen, also Personen mit Schnittstellenfunktion zu den VHS-Personalgruppen, für Inklusion sensibilisiert und als Multiplikatorinnen und Multiplikatoren installiert werden. Neben der expliziten Schaffung von Settings der Wissens- und Erfahrungsvermittlung werden auch bewährte Informations- und Austauschplattformen in Volkshochschulen wie Kursleitungskonferenzen genutzt: [56]

„[...] wir haben das aber aufgegriffen dann im Rahmen der Kursleiterkonferenz, dass wir das zum Tagesordnungspunkt gemacht haben und sie einfach darüber haben referieren lassen. Dass auch ein bisschen nochmal zusammengefasst haben, Handreichung ist, weiß Gott, zu viel gesagt, aber einfach eine Ideensammlung oder auch eine Tippsammlung, dass jemand, bei dem es wieder aufpoppt, dass wir da einfach nicht wieder bei null anfangen, sondern da mit einer Expertise auch weitergehen können“ (B27_HPM, Abs. 84). [57]

Hier kristallisiert sich eine weitere, in Volkshochschulen gängige Form der Wissens- und Erfahrungsvermittlung heraus: Die Expertisen Einzelner in Handreichungen oder Checklisten zu abstrahieren und als ein personenungebundenes Hilfsmittel Kursleitungen zur Verfügung zu stellen. Diese werden häufig in Ergänzung zu den unterschiedlichen Interaktionsformen eingesetzt und dienen der (Weiter-)Entwicklung und nachhaltigen Verstetigung inklusiver Handlungspraxis. [58]

Insgesamt weist die Fortbildungsförderung von Einzelplayern bzw. Kleingruppen zwei Perspektiven auf: Einerseits geht es um die Qualifizierung Einzelner, die im Bedarfsfall mit ihrem Wissen und ggf. ihrer Handlungspraxis für Austausch und Beratung zur Verfügung stehen (reaktiv). Andererseits hat die Fortbildungsförderung zum Ziel, geschulte Kursleitungen als Multiplikatorinnen und Multiplikatoren in der Breite (des jeweiligen Programmbereichs) einzusetzen und über geeignete Vermittlungsformate weiteres Lehrpersonal für Inklusion zu sensibilisieren (proaktiv). [59]

\section{Zusammenfassung und Perspektiven}

Die empirischen Teilergebnisse schlüsseln differenzierte Anstrengungen von Volkshochschulen für eine Fortbildungsförderung ihres Lehrpersonals auf: Die vier Dimensionen lassen sowohl auf eine reaktive als auch eine proaktive Handlungspraxis der Bedarfsdeckung bzw. Bedürfnisweckung schließen. Weiterführend bewegt sich die Umsetzung zwischen einer einrichtungsinternen und -externen Verortung sowie zwischen einzel- und gruppenbezogenen Maßnahmen. Diese sind weniger isoliert voneinander zu betrachten, sondern kommen vielmehr - in differenzierten Ausprägungsgraden - kombiniert in der aktuellen Fortbildungspraxis zum Einsatz. [60]

In einigen Untersuchungseinrichtungen lassen sich (in Ansätzen) Umsetzungsrealitäten einer Fortbildung für Inklusion erkennen, die eher selten einen ganzheitlichen und systematischen Charakter, jedoch Potenzial in eine solche Richtung aufweisen. Zumeist begegnet das Institutionalfeld konkreten Qualifikationsbedarfen, die im Kursgeschehen virulent werden. Kooperationseinrichtungen können dabei in unterschiedlichen Modi unterstützen. Im Zuge dessen kristallisiert sich der Regionsbezug als eine wesentliche Komponente heraus. Die Fortbildungsförderung kann insgesamt eine Bindungskraft für die Kursleitungen an die jeweilige Einrichtung besitzen - ein zentrales Moment für eine Organisation und ihre Entwicklungsoptionen für Inklusion bei einer Personalgruppe mit geringem Regulierungsgrad. [61]

Die allgemeine, öffentliche Erwachsenenbildung, die in ihren Angeboten, Prozessen und Strukturen bislang wenig inklusiv ausgerichtet ist und für Menschen mit Behinderung in der Regel nicht als Weiterbildungssetting wahrgenommen wird (Kapitel 1 \& 2), steht vor der 
zentralen Herausforderung, sich unter der Perspektive der Sicherung und Ausweitung gesellschaftlicher Partizipation fortzuentwickeln. Aufgrund der Spezifika der Institutionalstrukturen sowie der implizierten Beschäftigungsverhältnisse und Tätigkeitsausrichtungen der unterschiedlichen Personalgruppen zeigt sich der Ansatz einer organisationsgebundenen Professionalitätsentwicklung ${ }^{8}$ anschlussfähig (Schicke, 2012; Seitter, 2011). „Dieses Verständnis fokussiert Professionalität(sentwicklung) vor allem als ein wechselseitiges, relationales Verschränkungsverhältnis von Profession und Organisation(sentwicklung) mit einer starken Betonung der Standortgebundenheit der konkreten community of practise vor Ort" (LauberPohle \& Seitter, 2020, Abs. 33, Hervorhebung im Original). Im Zuge dessen wird Professionalität kontinuierlich rekonstruiert und situativ hergestellt; ein routinisiertes Handeln oder eine organisationale Überführung sind daher kaum möglich. Nittel (2000) definiert wie folgt: [62]

\begin{abstract}
„Professionalität ist, so kann man zusammenfassend sagen, kein ,Zustand', der errungen oder erreicht werden kann, sondern eine flüchtige, jedes Mal aufs Neue situativ herzustellende berufliche Leistung. Sie kann weder verordnet werden, noch erschöpft sie sich in der Ausformulierung normativer Prämissen. Professionalität stellt in dieser Perspektive somit ein extrem störanfälliges, durch das Merkmal der Fallibilität gekennzeichnetes Handlungsphänomen dar" (Nittel, 2000, S. 85). [63]
\end{abstract}

Im Kontext des Lernfeldes Inklusion und den damit verknüpften Anforderungen an Qualifikation stellt sich die Frage, wie Lehrende als zentrale Repräsentantinnen und Repräsentanten von Volkshochschulen für eine inklusive Angebotsgestaltung befähigt werden können. Die Ermöglichung unterschiedlicher Fortbildungsmodi kann dabei eine Handlungsoption darstellen. Lauber-Pohle und Seitter (2020) richten einen netzwerkanalytischen Blick auf inklusionsorientierte Bildungsarrangements und fassen hinsichtlich des Bezugsfeldes von Blindheit und Sehbeeinträchtigung als eine Form von Sinnesbeeinträchtigung zusammen: [64]

\begin{abstract}
„Bezogen auf die Entwicklung inklusiver erwachsenenpädagogischer Fachlichkeit bei Blindheit und Sehbeeinträchtigung würde diese Perspektive eine stärkere Rückbindung an die je konkreten organisationalen Voraussetzungen und Kontextbedingungen für blinden- und sehbehinderungsspezifische Öffnungen implizieren und die Arbeit an den verschiedenen Dimensionen von Barrierefreiheit, Zugänglichkeit und Adaptivität als eine Verschränkung von professions- und organisationsbezogenen Aufgaben und Abläufen konzipieren“ (Lauber-Pohle \& Seitter, 2020, Abs. 33). [65]
\end{abstract}

Aufgrund der höchst unterschiedlichen Assistenzbedarfe und Teilnahmebarrieren von Menschen mit Beeinträchtigung sowie ihren Möglichkeiten von Lernen und Handeln oder Erleben und Erfahren bieten sich daher sowohl Formate im Sinne einer allgemeinen Sensibilisierung für Inklusion als auch einer Auseinandersetzung mit spezifischer Beeinträchtigung an. [66]

\footnotetext{
1 Im vorliegenden Beitrag werden die Begriffe der Erwachsenenbildung und Weiterbildung synonym für alle öffentlichen Angebote des quartären Bildungsbereichs zur Fortsetzung oder Wiederaufnahme organisierten Lernens verwendet (Süssmuth \& Eisfeld, 2018, S. 764).

2 Das Vorhaben wird von Dezember 2017 bis Februar 2021 vom Bundesministerium für Bildung und Forschung (BMBF) im Rahmen der Richtlinie „Qualifizierung der pädagogischen Fachkräfte für inklusive Bildung" unter dem Förderkennzeichen 01NV1714 gefördert. Zu den Kooperationspartnern zählen der Hessische Volkshochschulverband e.V. (hvv), der Deutsche Verein der Blinden und Sehbehinderten in Studium und Beruf e.V. (DVBS), der Deutsche Blinden- und Sehbehindertenverband e.V. (DBSV) sowie die Deutsche Blindenstudienanstalt e.V. (blista). Weitere Projektinformationen sind zu finden unter: http://uni-marburg.de/TEVUv.

3 „Blindheit als diejenige Bedingung, die in der Auseinandersetzung mit der materialen und sozialen Umwelt nicht auf Visualität Bezug nehmen kann, wird unterschieden von Sehbeeinträchtigung als
} 
derjenigen Bedingung, deren Auseinandersetzung mit der materialen und sozialen Umwelt auf eine wie auch immer geartete Visualität bezogen ist" (Walthes, 2014, S. 18f., Hervorhebung im Original).

4 Die Praxispartner unterstützen das Vorhaben sowohl im Rahmen des Feldzugangs der Befragungen und der Barrierefreiheit der Erhebungsinstrumente als auch im Rahmen der Entwicklung der Fortbildungsreihen und der Veröffentlichung von Handreichungen.

${ }^{5}$ Eine Auseinandersetzung mit dem Begriff der Zielgruppe und seinen Dimensionen findet sich exemplarisch bei Schiersmann (2010).

6 Eine Auseinandersetzung mit Volkshochschulen als lernende Organisationen findet sich exemplarisch bei Feld (2007).

7 Eine Differenzierung der unterschiedlichen Modi von Kooperationsbeziehungen auf struktureller Ebene findet sich exemplarisch bei Seitter (2013).

8 Das Verständnis von einer organisationsbezogenen bzw. organisationsgebundenen Professionalität ist vor allem auf Schäffter (1998) zurückzuführen, der Professionalität als eine komplexe Gesamtleistung einer Organisation aus einer Mehrebenenperspektive heraus betrachtet.

\section{Literatur}

Ackermann, K.-E. (2017). „Pädagogische Professionalität“ im Handlungsfeld inklusive Erwachsenenbildung. Eine Problemskizze in vier Thesen. In C. Lindmeier \& H. Weiß (Hrsg.), Pädagogische Professionalität im Spannungsfeld von sonderpädagogischer Förderung und inklusiver Bildung (S. 134-152). Sonderpädagogische Förderung heute, 1. Beiheft. Weinheim \& Basel: Beltz Juventa.

Ackermann, K.-E., Burtscher, R., Ditschek, E. J. \& Schlummer, W. (Hrsg.). (2012). Inklusive Erwachsenenbildung. Kooperationen zwischen Einrichtungen der Erwachsenenbildung und der Behindertenhilfe. Berlin: Gesellschaft Erwachsenenbildung und Behinderung.

Autorengruppe Bildungsberichterstattung. (2014). Bildung in Deutschland 2014. Ein indikatorengestützter Bericht mit einer Analyse zur Bildung von Menschen mit Behinderungen. Verfügbar unter https://www.bildungsbericht.de/de/bildungsberichte-seit-2006/bildungsbericht-2014/pdf-bildungsbericht-2014/bb-2014.pdf

Autorengruppe Bildungsberichterstattung. (2018). Bildung in Deutschland 2018. Ein indikatorengestützter Bericht mit einer Analyse zu Wirkungen und Erträgen von Bildung. Verfügbar unter https://www.bildungsbericht.de/de/bildungsberichte-seit-2006/bildungsbericht-2018/pdf-bildungsbericht-2018/bildungsbericht-2018.pdf

Babilon, R. (2018). Inklusive Erwachsenenbildung mit Menschen mit Lernschwierigkeiten - eine qualitative Studie in England. Universität Koblenz-Landau, Landau. Verfügbar unter https://kola.opus.hbz-nrw.de/opus45-kola/frontdoor/deliver/index/docld/1682/file/Babilon_ Inklusive+Erwachsenenbildung_Dissertation.pdf

Babilon, R., Weiß, M. \& Brandt, P. (2019). »Inklusion muss nicht begründet werden«. weiter bilden spricht mit Rebecca Babilon und Michael Weiß. DIE Zeitschrift für Erwachsenenbildung, 26(1), 12-16. doi: 10.3278/WBDIE1901W012

Burtscher, R., Ditschek, E. J., Ackermann, K.-E., Kil, M. \& Kronauer, M. (Hrsg.). (2013). Zugänge zu Inklusion. Erwachsenenbildung, Behindertenpädagogik und Soziologie im Dialog. Bielefeld: W. Bertelsmann Verlag. doi: 10.3278/14/1114w

Deutsches Zentrum für Altersfragen. (2016). Deutscher Alterssurvey 2014. Zentrale Befunde. Verfügbar unter https://www.bmfsfj.de/blob/jump/77122/deutscher-alterssurvey-2014-vielfalt-und-wandel-des-alters-zentrale-befunde-data.pdf

Döbert, H. \& Weishaupt, H. (2013). Einleitung. In H. Döbert \& H. Weishaupt (Hrsg.), Inklusive Bildung professionell gestalten. Situationsanalyse und Handlungsempfehlungen (S. 7-10). Münster: Waxmann Verlag. 
Feld, T. C. (2007). Volkshochschulen als „lernende Organisationen“. Entwicklung eines Anforderungsprofils unter Berücksichtigung theoretischer Ansätze organisationalen Lernens sowie einer innerorganisationalen und einer außerorganisationalen Perspektive. Hamburg: Verlag Dr. Kovač.

Furrer, H. (2013). Didaktische und methodische Überlegungen zur Inklusion in der Erwachsenenbildung. In R. Burtscher, E. J. Ditschek, K.-E. Ackermann, M. Kil \& M. Kronauer (Hrsg.), Zugänge zu Inklusion. Erwachsenenbildung, Behindertenpädagogik und Soziologie im Dialog (S. 197-208). Bielefeld: W. Bertelsmann Verlag.

Gieseke, W. (2018). Professionalität und Professionalisierung in der Erwachsenenbildung/ Weiterbildung. In R. Tippelt \& A. von Hippel (Hrsg.), Handbuch Erwachsenenbildung/Weiterbildung (6. Aufl., S. 1051-1069). Wiesbaden: Springer VS.

Harmeier, M. (2009). „Für die Teilnehmer sind wir die VHS“. Selbstverständnis von Kursleitenden und ihr Umgang mit Qualifizierungsmaßnahmen. Bielefeld: W. Bertelsmann Verlag.

Hessischer Volkshochschulverband e.V. (Hrsg.) (2019). Inklusive Erwachsenenbildung. Hessische Blätter für Volksbildung, 69(1). doi: 10.3278/HBV1901W

Hippel, A. von \& Tippelt, R. (Hrsg.). (2009). Fortbildung der Weiterbildner/innen. Eine Analyse der Interessen und Bedarfe aus verschiedenen Perspektiven. Weinheim \& Basel: Beltz Verlag.

Hirschberg, M. \& Lindmeier, C. (2013). Der Begriff „Inklusion“ - Ein Grundsatz der Menschenrechte und seine Bedeutung für die Erwachsenenbildung. In R. Burtscher, E. J. Ditschek, K.E. Ackermann, M. Kil \& M. Kronauer (Hrsg.), Zugänge zu Inklusion. Erwachsenenbildung, Behindertenpädagogik und Soziologie im Dialog (S. 39-52). Bielefeld: W. Bertelsmann Verlag.

Korb, C. A., Kottler, U. B., Wolfram, C., Hoehn, R., Schulz, A., Zwiener, I. et al. (2014). Prevalence of age-related macular degeneration in a large European cohort: Results from the population-based Gutenberg Health Study. Graefe's Archive for Clinical and Experimental Ophthalmology, 252(9), 1403-1411.

Kraft, S., Seitter, W. \& Kollewe, L. (2009). Professionalitätsentwicklung des Weiterbildungspersonals. Bielefeld: W. Bertelsmann Verlag. doi: 10.3278/81/0101w

Kuckartz, U. (2018). Qualitative Inhaltsanalyse. Methoden, Praxis, Computerunterstützung (4. Aufl.). Weinheim \& Basel: Beltz Juventa.

Lauber-Pohle, S. (2019). Dimensionen einer inklusiven, allgemeinen Erwachsenenbildung. Hessische Blätter für Volksbildung, 69(1), 7-17. doi: 10.3278/HBV1901W

Lauber-Pohle, S. \& Seitter, W. (2020). Erwachsenenpädagogische Fachlichkeit für eine inklusive allgemeine Erwachsenenbildung: eine kooperative Mehrebenenherausforderung am Beispiel von Blindheit und Sehbeeinträchtigung. Qfl - Qualifizierung für Inklusion, 2(1). doi: 10.21248/qfi.29

Martin, A., Lencer, S., Schrader, J., Koscheck, S., Ohly, H., Dobischat, R. et al. (2016). Das Personal in der Weiterbildung. Arbeits- und Beschäftigungsbedingungen, Qualifikationen, Einstellungen zu Arbeit und Beruf. Bielefeld: W. Bertelsmann Verlag.

Möller, S. (2011). Marketing in der Erwachsenenbildung. Bielefeld: W. Bertelsmann Verlag. doi: 10.3278/42/0031w

Münchner Volkshochschule. (2015). Münchner Erklärung zur Inklusion und öffentlich verantworteten Erwachsenenbildung. Verfügbar unter https://www.mvhs.de/programm/ muenchner-erklaerung-zur-inklusion-und-oeffentlich-verantworteten-erwachsenenbildung

Nittel, D. (2000). Von der Mission zur Profession? Stand und Perspektiven der Verberuflichung in der Erwachsenenbildung. Bielefeld: W. Bertelsmann Verlag.

Reichart, E., Huntemann, H. \& Lux, T. (2020). Volkshochschul-Statistik. 57. Folge, Berichtsjahr 2018 (2. Aufl.). doi: 10.3278/85/0022aw

Schäffter, O. (1998). Weiterbildung in der Transformationsgesellschaft. Zur Grundlegung einer Theorie der Institutionalisierung. Berlin: Arbeitsgemeinschaft Qualifikations-EntwicklungsManagement, Geschäftsstelle der Arbeitsgemeinschaft betriebliche Weiterbildung e.V. 
Schicke, H. (2012). Organisation als Kontext der Professionalität. Beruflichkeit pädagogischer Arbeit in der Transformationsgesellschaft. Bielefeld: W. Bertelsmann Verlag. doi: 10.3278/ 14/1111w

Schiersmann, C. (2010). Zielgruppen. In R. Arnold, S. Nolda \& E. Nuissl (Hrsg.), Wörterbuch Erwachsenenpädagogik (S. 321-323). Bad Heilbrunn: Klinkhardt /UTB.

Seitter, W. (2009). Professionalitätsentwicklung als aufgabenbezogene Tätigkeitserweiterung und berufsbiographische Kompetenzaufschichtung: Ein Aufriss. In W. Seitter (Hrsg.), Professionalitätsentwicklung in der Weiterbildung (S. 11-16). Wiesbaden: VS Verlag für Sozialwissenschaften.

Seitter, W. (2011). Wandel des Professionellen im Feld der Weiterbildung. In W. Helsper \& R. Tippelt (Hrsg.), Pädagogische Professionalität (S. 122-137). Zeitschrift für Pädagogik, 57. Beiheft. Weinheim \& Basel: Beltz Verlag.

Seitter, W. (2013). Professionelles Handeln im Kooperations- und Vernetzungskontext. In K. Dollhausen, T. C. Feld \& W. Seitter (Hrsg.), Erwachsenenpädagogische Kooperations- und Netzwerkforschung (S. 33-48). Wiesbaden: Springer VS.

Seitter, W. \& Franz, J. (2019). Inklusive Erwachsenenbildung. Hessische Blätter für Volksbildung, 69(1), 3-6. doi: 10.3278/HBV1901W

Süssmuth, R. \& Eisfeld, K. H. (2018). Volkshochschule. Erwachsenenbildung/Weiterbildung in öffentlicher Verantwortung. In R. Tippelt \& A. von Hippel (Hrsg.), Handbuch Erwachsenenbildung/Weiterbildung (6. Aufl., S. 763-784). Wiesbaden: Springer VS.

Walthes, R. (2014). Einführung in die Pädagogik bei Blindheit und Sehbeeinträchtigung (3. Aufl.). München: Ernst Reinhardt.

\section{Kontakt}

Simone Krähling, Philipps-Universität Marburg, Institut für Erziehungswissenschaft, Wilhelm-RöpkeStraße 6A, 35039 Marburg

E-Mail: simone.kraehling@uni-marburg.de

\section{Zitation}

Krähling, S. (2020). Professionalitätsentwicklung in der allgemeinen, öffentlichen Erwachsenenbildung für Inklusion - ein Systematisierungsversuch der Fortbildungspraxis an Volkshochschulen. Qfl - Qualifizierung für Inklusion, 2(4), doi: 10.21248/Qfl.49

Eingereicht: 17 . Mai 2020

Veröffentlicht: 17. Dezember 2020

\section{(cc) BY-ND}

Dieser Text ist lizenziert unter einer Creative Commons Namensnennung - Keine Bearbeitungen 4.0 International Lizenz. 